\title{
Dynamics of an ion chain in a harmonic potential
}

\author{
Giovanna Morigi ${ }^{1}$ and Shmuel Fishman ${ }^{2}$ \\ ${ }^{1}$ Department of Quantum Physics, University of Ulm, Albert-Einstein-Allee 11, D-89069 Ulm, Germany \\ ${ }^{2}$ Department of Physics, Technion, 32000 Haifa, Israel \\ (Received 14 May 2004; revised manuscript received 26 October 2004; published 28 December 2004)
}

\begin{abstract}
Cold ions in anisotropic harmonic potentials can form ion chains of various sizes. Here, the density of ions is not uniform, and thus the eigenmodes are not phononic-like waves. We study chains of $N \gg 1$ ions and evaluate analytically the long-wavelength modes and the density of states in the short-wavelength limit. These results reproduce with good approximation the dynamics of chains consisting of dozens of ions. Moreover, they allow one to determine the critical transverse frequency required for the stability of the linear structure, which is found to be in agreement with results obtained by different theoretical methods [D. H. E. Dubin, Phys. Rev. Lett. 71, 2753 (1993)] and by numerical simulations [J. P. Schiffer, Phys. Rev. Lett. 70, 818 (1993)]. We introduce and explore the thermodynamic limit for the ion chain. The thermodynamic functions are found to exhibit deviations from extensivity.
\end{abstract}

DOI: 10.1103/PhysRevE.70.066141

PACS number(s): 05.20.-y, 61.50.-f, 52.27.Jt, 32.80.Pj

\section{INTRODUCTION}

Coulomb crystals are organized structures of charged particles, which interact through the Coulomb repulsion and organize in regular patterns at sufficiently low temperatures in presence of a confining potential [1]. These potentials are realized by means of Paul or Penning traps [2], and their geometry determines the crystal's structure. Several remarkable experiments have reported crystallization of ion gases in Paul traps [3-7], Penning traps [8], and ion-storage rings [9]. The Bragg scattering in three-dimensional structures was studied, providing information about the internal structure of the crystal $[10,11]$. These crystals represent a kind of rarefied condensed matter, the interparticle distance being of the order of several micrometers, allowing to study the structure by means of optical radiation. Variation of the potential permits one to control the crystal shape as well as the number of ions, thus offering the unique opportunity to study the transition from few particles to mesoscopic systems. Besides, these structures have been the object of growing interest as they provide promising applications for spectroscopy $[12,13]$, frequency standards [14], study and control of chemical reactions [15], and quantum information processors [16-19].

In this work we investigate the dynamics of Coulomb chains. These are one-dimensional structures, obtained by means of strong transverse confinement and which usually consist of dozens of ions localized along the trap axis $[5,6]$. They represent a peculiar crystallized structure: In fact, due to the axial potential, the equilibrium charge distribution is not uniform [20,21]. This is in contrast to the threedimensional case, where the density of charges in a harmonic potential is uniform and, therefore, where the eigenmodes are phononic-like waves. In the Coulomb chain the nonuniformity of the density of ions combined with the long-range interaction results in excitations that are fundamentally different from the phonons in solids and leads to interesting thermodynamic properties. The exploration of these excitations and of the chain thermodynamics is the subject of the present paper, which extends and complements the results presented in [22].
Our starting point is the ions equilibrium configuration evaluated in [20]. We investigate the dynamics for small oscillations, when the harmonic approximation is valid, in the limit of a large number of ions. We derive analytically the eigenfrequencies and the corresponding eigenmodes for the long-wavelength excitations. These are compared with numerical results and good agreement is found. From the resulting dispersion relation the density of states of the longwavelength eigenmodes is determined. An analytic form of the density of states is also found for the short-wavelength excitations. This result allows one to evaluate the critical aspect ratio between the frequencies of the transverse and axial confining potential, which determines the stability of the chain. The value we find agrees with numerical results [23], which have been experimentally verified for chains of a few ions [24]. In particular, it is in agreement with the analytical estimate in [25], which was obtained under different requirements.

Using these results we discuss the statistical mechanics of the chain and derive some thermodynamic quantities in a specific thermodynamic limit, which is defined here by keeping constant the density of ions in the chain center, as the number of ions tends to infinity and the axial frequency to zero, analogously to the definition for cold neutral gases in traps [26]. Nonextensive thermodynamic properties are found. We compare the thermodynamic functions with the ones of a chain of a finite number of ions that are obtained numerically and find reasonable agreement.

This work is organized as follows. In Sec. II we introduce the basic equations and discuss the fundamental properties. In Sec. III the spectrum of excitations is studied. In Sec. IV we investigate the statistical mechanics of the system. Section V presents the conclusions and outlook. In the Appendixes, several details of the calculations of Sec. III are reported.

\section{STRING OF CHARGES IN A HARMONIC POTENTIAL}

The Hamiltonian describing the dynamics of a chain of $N$ ions of mass $m$ and charge $Q$, which are confined by a harmonic potential, is given by 


$$
H=\sum_{j=1}^{N} \frac{\mathbf{p}_{j}^{2}}{2 m}+V\left(\mathbf{r}_{1}, \ldots, \mathbf{r}_{N}\right)
$$

where $\mathbf{r}_{j}=\left(x_{j}, y_{j}, z_{j}\right)$ and $\mathbf{p}_{j}$ are the positions and conjugate momenta $(j=1, \ldots, N)$. The term $V$ accounts for the oscillator's potential and the Coulomb repulsion,

$$
\begin{aligned}
V= & \frac{1}{2} \sum_{j} m\left[\nu^{2} x_{j}^{2}+\nu_{t}^{2}\left(y_{j}^{2}+z_{j}^{2}\right)\right] \\
& +\frac{1}{2} \sum_{j=1}^{N} \sum_{j \neq i} \frac{Q^{2}}{\sqrt{\left(x_{i}-x_{j}\right)^{2}+\left(y_{i}-y_{j}\right)^{2}+\left(z_{i}-z_{j}\right)^{2}}},
\end{aligned}
$$

where the harmonic oscillator has rotational symmetry around the $x$ axis with axial and transverse frequencies $\nu$ and $\nu_{t}$, respectively.

For sufficiently low kinetic energy crystallization occurs. The temperature at which the gas is crystallized corresponds to large plasma parameters $\Gamma=Q^{2} / a_{\mathrm{WS}} k_{B} T \gg 1$. Here, $a_{\mathrm{WS}}$ is the Wigner-Seitz radius, which is a function of the mean density $n$, and is defined as $a_{\mathrm{WS}}=(3 / 4 \pi n)^{1 / 3}$ [1]. In this regime the ions are localized at the classical equilibrium positions $\mathbf{r}_{j}^{(0)}$, which satisfy the equations $\partial V / \partial \mathbf{r}_{j} \mid \mathbf{r}_{j}^{(0)}=\mathbf{0}$, and such that the potential energy is minimal. When the harmonic potential is sufficiently asymmetric-i.e., for $\nu \ll \nu_{t}$ - the ion equilibrium positions are confined to the trap axis [23], namely, $\mathbf{r}_{j}^{(0)}=\left(x_{j}^{(0)}, 0,0\right)$, and satisfy the equation describing the equilibrium of the forces:

$$
m \nu^{2} x_{i}^{(0)}=-\sum_{j>i} \frac{Q^{2}}{\left(x_{j}^{(0)}-x_{i}^{(0)}\right)^{2}}+\sum_{j<i} \frac{Q^{2}}{\left(x_{i}^{(0)}-x_{j}^{(0)}\right)^{2}},
$$

where the numbering convention is $x_{i}>x_{j}$ for $i>j$. The stability of these points with respect to the transverse vibrations depends on the number of ions, $N$, and on the ratio $\nu_{t} / \nu$, and it is discussed in Sec. III B. In this section, we assume that the configuration is stable and approximate the potential by its second-order Taylor expansion around the points $\mathbf{r}_{j}^{(0)}$. We denote by $q_{i}=x_{i}-x_{i}^{(0)}$ the displacements in the $\hat{x}$ direction and approximate the Hamiltonian (1) as

$$
H \approx V_{0}+H_{\text {har }}
$$

where $V_{0}=V\left(\mathbf{r}_{1}^{(0)}, \ldots, \mathbf{r}_{N}^{(0)}\right)$ is the classical minimum energy, while $H_{\text {har }}$ describes the (classical) harmonic oscillations around the equilibrium points [27-29],

$$
\begin{aligned}
H_{\mathrm{har}}= & \sum_{j=1}^{N} \frac{\mathbf{p}_{j}^{2}}{2 m}+\frac{1}{2} \sum_{j} m \nu^{2} q_{j}^{2}+\frac{1}{2} \sum_{j} m \nu_{t}^{2}\left(y_{j}^{2}+z_{j}^{2}\right) \\
& +\frac{1}{4} \sum_{i} \sum_{j \neq i} K_{i, j}\left(q_{i}-q_{j}\right)^{2} \\
& -\frac{1}{8} \sum_{i} \sum_{j \neq i} K_{i, j}\left[\left(y_{i}-y_{j}\right)^{2}+\left(z_{i}-z_{j}\right)^{2}\right]
\end{aligned}
$$

and the coefficients $K_{i, j}=\partial^{2} V /\left.\partial x_{j}^{2}\right|_{\left\{x_{l}^{(0)}\right\}}$ are positive and take the form

$$
K_{i, j}=\frac{2 Q^{2}}{\left|x_{i}^{(0)}-x_{j}^{(0)}\right|^{3}} .
$$

Equation (4) shows that the axial motion is decoupled from the transverse motion in the harmonic expansion. The corresponding equations of motion are

$$
\begin{aligned}
& \ddot{q}_{i}=-\nu^{2} q_{i}-\sum_{j \neq i} \frac{K_{i, j}}{m}\left(q_{i}-q_{j}\right), \\
& \ddot{y}_{i}=-\nu_{t}^{2} y_{i}+\frac{1}{2} \sum_{j \neq i} \frac{K_{i, j}}{m}\left(y_{i}-y_{j}\right), \\
& \ddot{z}_{i}=-\nu_{t}^{2} z_{i}+\frac{1}{2} \sum_{j \neq i} \frac{K_{i, j}}{m}\left(z_{i}-z_{j}\right),
\end{aligned}
$$

and describe a system of coupled oscillators, with long-range interaction and position-dependent coupling strength. In this paper eigenmodes will be calculated. For this we assume $q_{i}(t)=\int \mathrm{e}^{\mathrm{i} \omega t} \widetilde{q}_{i}(\omega) d \omega / 2 \pi$. To simplify the notation we replace $\tilde{q}_{i}(\omega)$ by $q_{i}$. This results in equations for the eigenmodes of frequency $\omega$ that are similar to Eq. (6), but with $\ddot{q}_{i}$ replaced by $-\omega^{2} q_{i}$. The same replacement will be performed for $y_{i}(t)$ and $z_{i}(t)$.

It can be easily verified that the center-of-mass motion is an eigenmode of the secular equations (6)-(8). The axial center-of-mass mode is $q_{1}=\cdots=q_{N}$ at the characteristic frequency $\nu$, while the transverse center-of-mass modes are $y_{1}$ $=\cdots=y_{N}$ and $z_{1}=\cdots=z_{N}$ at frequency $\nu_{t}$. We remark that the axial and transverse coupling terms appearing in Eqs. (6)-(8) have opposite signs. Due to this property, in the axial direction the collective excitations are of higher frequencies than the center-of-mass frequency $\nu$, while in the transverse plane the collective excitations are of lower frequencies than $\nu_{t}$.

\section{Properties and symmetries}

The Hamiltonian (4) is not translationally invariant, and this is a consequence of the nonuniformity of the ions equilibrium distribution, due to the harmonic force appearing in Eq. (3). The Hamiltonian (4) is, however, invariant under reflection with respect to the center of the trap, which coincides with the origin of the axes. In particular,

$$
x_{i}^{(0)}=-x_{-i}^{(0)},
$$

where $i=1, \ldots, N^{\prime}$ [here, $N^{\prime}=N / 2$ for even $N$, while $N^{\prime}$ $=(N-1) / 2$ for odd $N]$. Hence, the normal modes of the chain are symmetric (even) or antisymmetric (odd) under reflection with respect to the center [28,29], such that

$$
w_{i}^{(n)}= \pm w_{-i}^{(n)},
$$

with $w_{i}^{(n)}=q_{i}^{(n)}, y_{i}^{(n)}, z_{i}^{(n)}$ and $n$ labels the mode. Some general properties can be inferred from this simple consideration. For instance, the even modes of the axial motion are characterized by constant length $L$ of the chain, since $q_{N^{\prime}}^{(n)}=q_{-N^{\prime}}^{(n)}$. For the odd modes, on the other hand, the center of mass of the chain, which coincides with the chain center, does not move. 
Clearly, the center-of-mass mode, which we denote by $w_{i}^{(1)}$, is an even mode characterized by equal displacements at the positions $x_{i}^{(0)}$ of the chain. This property and the orthogonality between the normal modes lead to the relation

$$
\sum_{j} w_{j}^{(n)}=0
$$

for all normal modes with $n>1$.

It is remarkable that also the lowest axial odd mode (stretch mode) and its frequency can be exactly determined. In fact, taking $q_{i}^{(2)} \propto x_{i}^{(0)}$ and substituting into Eq. (6) one finds

$$
\begin{aligned}
\left(\omega^{2}-\nu^{2}\right) q_{i}^{(2)} & =-\sum_{j>i} \frac{2 Q^{2} / m}{\left(x_{j}^{(0)}-x_{i}^{(0)}\right)^{2}}+\sum_{j<i} \frac{2 Q^{2} / m}{\left(x_{i}^{(0)}-x_{j}^{(0)}\right)^{2}} \\
& =2 \nu^{2} q_{i}^{(2)},
\end{aligned}
$$

where we have used relation (3). Therefore, the frequency of the axial stretch mode $q_{i}^{(2)}$ is $\sqrt{3} \nu$ and its value is independent of the number of ions $N$ of the chain. This property was first demonstrated in [30]. It has also been observed by numerical evaluation of the normal modes of chains up to ten ions $[17,27]$. Analogously, the transverse stretch mode, which is the highest odd transverse excitation, satisfies $y_{i}^{(2)}, z_{i}^{(2)} \propto x_{i}^{(0)}$ with eigenfrequency $\sqrt{\nu_{t}^{2}-\nu^{2}}$, which is also independent of $N$.

We remark that the invariance under reflection imposes different boundary conditions than the ones that are usually chosen for a crystal with uniform ion distribution. In a crystal that is translationally invariant even and odd modes are degenerate and one may choose periodic boundary conditions [31]. In the presence of an external potential with central symmetry this invariance is broken, apart for the mirror symmetry with respect to the center. Hence, at the edges the eigenmodes fulfill the relation $w_{-N^{\prime}}= \pm w_{N^{\prime}}$, where the sign is determined by the parity.

\section{SECULAR PROBLEM}

The systematic derivation of an analytic solution of Eqs. (6)-(8) is a challenging problem, since it requires one to take systematically into account the position-dependent coupling constant and the long-range interaction. Nevertheless, in the limit of a large number of ions, $N \gg 1$, we can make some simplifying assumptions. In this limit, in fact, the interparticle spacing $a_{L}\left(x_{i}\right)=x_{i+1}^{(0)}-x_{i}^{(0)}$ is a smooth function of the position, and it is inversely proportional to the density of ions per unit length [20],

$$
n_{L}\left(x_{i}\right)=1 / a_{L}\left(x_{i}\right) .
$$

The density of charges for unit length can be evaluated by applying the Gauss theorem to a continuous distribution of charges, which is assumed to be uniformly distributed in an elongated ellypsoid. The resulting one dimensional density is [25]

$$
n_{L}(x)=\frac{3}{4} \frac{N}{L}\left(1-\frac{x^{2}}{L^{2}}\right),
$$

which is defined for $|x| \leqslant L$, where $2 L$ is the length of the crystal at equilibrium. The density (12) is the leading term in the expansion in powers of $1 / \ln N$ and it gives a good estimate of the charge distribution in the center of the chain for $N$ sufficiently large [20]. The length $L$ is evaluated by minimizing the energy of the crystal and at leading order in $\ln N$ fulfills the relation [20]

$$
L(N)^{3}=3\left(\frac{Q^{2}}{m \nu^{2}}\right) N \ln N
$$

In the following, we use these quantities to derive an approximate solution for the long- and short-wavelength modes in the limit of $N \gg 1$ ions. Furthermore, we compare the results of the derivation with the numerical calculations, which determine the spectrum by solving Eqs. (3) and then diagonalizing the matrix determining the eigenvalue equations (6)-(8) for a finite number of ions.

\section{A. Eigenmodes in the long-wavelength limit}

We use the ansatz $q_{i}(x, t)=\mathrm{e}^{\mathrm{i} \omega t} \widetilde{q}_{i}(x)$ in Eq. (6) and define the rescaled positions $\xi_{i}^{(0)}=x_{i}^{(0)} / L$ and the rescaled interparticle distances $a\left(\xi_{i}\right)=a_{L}\left(x_{i}\right) / L$. With these definitions, denoting for simplicity of notation $\widetilde{q}_{i} \rightarrow q_{i}$, Eq. (6) takes the form

$$
\left(\omega^{2}-\nu^{2}\right) q_{i}=\nu^{2} \mathcal{K}_{0} \sum_{j \neq i} \frac{1}{\left|\xi_{i}^{(0)}-\xi_{j}^{(0)}\right|^{3}}\left(q_{i}-q_{j}\right),
$$

where we have introduced the dimensionless constant

$$
\mathcal{K}_{0}=\frac{2 Q^{2}}{m \nu^{2} L(N)^{3}}=\frac{2}{3 N \ln N} .
$$

If the number of ions is large $(N \gg 1)$, for the longwavelength modes one can approximate the chain by a continuous distribution of charges. In this limit, $\xi$ is a continuous variable varying in the interval $(-1,1)$, while the displacement $q_{i}=q\left(\xi_{i}\right)$ is a continuous function, here denoted by $q(\xi)$. Then, Eq. (14) takes the form

$$
\left(\omega^{2}-\nu^{2}\right) q(\xi)=\frac{3}{4} \nu^{2} \mathcal{K}_{0} N I(\xi, q(\xi)),
$$

where

$$
\begin{aligned}
I(\xi, q(\xi))= & \int_{-1}^{\xi-a(\xi)} \mathrm{d} \xi^{\prime} \frac{n\left(\xi^{\prime}\right)}{\left(\xi-\xi^{\prime}\right)^{3}}\left[q(\xi)-q\left(\xi^{\prime}\right)\right] \\
& +\int_{\xi+a(\xi)}^{1} \mathrm{~d} \xi^{\prime} \frac{n\left(\xi^{\prime}\right)}{\left(\xi^{\prime}-\xi\right)^{3}}\left[q(\xi)-q\left(\xi^{\prime}\right)\right],
\end{aligned}
$$

while $n(\xi)=1-\xi^{2}$ is the density of charges normalized to $4 / 3$. Equations (16) and (17) are valid away from the edges of the chain and for long-wavelength excitations, where the continuum approximation is reasonable. The continuum limit for Eq. (7) gives 


$$
\left(\nu_{t}^{2}-\omega^{2}\right) y(\xi)=\frac{3}{8} \nu^{2} \mathcal{K}_{0} N I(\xi, y(\xi))
$$

A similar type of equations is obtained for $z_{i}$. It is remarkable that the axial trap frequency enters only as a prefactor on the right-hand side of Eq. (16). Consequently the axial eigenfrequencies are proportional to $\nu$. The transverse eigenfrequencies, instead, do not show this behavior, as one can see from Eq. (18): Here, it is the quantity $\nu_{t}^{2}-\omega^{2}$ which is proportional to $\nu^{2}$. The proportionality constants depend on the modes and will be calculated in what follows within some approximations. The results are independent of the charge $Q$ and of the mass $m$.

According to Eqs. (16)-(18), the secular problem consists of solving the eigenvalue equation

$$
I\left(\xi, w^{(n)}(\xi)\right)=\lambda_{n} w^{(n)}(\xi),
$$

where $w^{(n)}(\xi)$ can be the axial or transverse mode, which satisfies the orthogonality relation

$$
\int_{-1}^{1} \mathrm{~d} \xi n(\xi) w^{(n)}(\xi) * w^{(m)}(\xi)=\delta_{n, m}
$$

By partial integration Eq. (17) can be written as the sum of two terms,

$$
I=I_{0}+\Delta I,
$$

where $I_{0}$ contains the contributions of the ions around the point $\xi$, while the term $\Delta I$ is determined by the value of the density and of the eigenmode function $q(\xi)$ and their derivatives at the end-points of the chain. In Appendix A we derive the explicit form of the two terms and discuss their order of magnitude. For the long-wavelength modes and at the points $\xi$ sufficiently far away from the chain end-points $\xi= \pm 1$, we find $I \approx I_{0}$ where

$$
\begin{aligned}
I_{0}(\xi, w(\xi))= & \left(\ln a(\xi)-\frac{3}{2}\right)\left[n(\xi) w^{\prime \prime}(\xi)+2 n^{\prime}(\xi) w^{\prime}(\xi)\right] \\
& +o(a(\xi))
\end{aligned}
$$

and $a(\xi)$ is an infinitesimal quantity of the order $1 / N$. Using Eqs. (11) and (12) in Eq. (21) we obtain

$$
I_{0}(\xi, w(\xi))=-\left(\ln N+\ln \frac{3}{4}+\ln \left(1-\xi^{2}\right)+\frac{3}{2}\right) J(w(\xi)),
$$

where

$$
J(w(\xi))=\left(1-\xi^{2}\right) w^{\prime \prime}(\xi)-4 \xi w^{\prime}(\xi) .
$$

In the limits of validity of Eq. (21) and for $N$ sufficiently large, such that $\ln N \gg 1$, Eq. (22) can be approximated by the leading order in $\ln N$,

$$
I_{0}(\xi, w(\xi)) \approx-\ln N J(w(\xi)),
$$

and the eigenvalue equation (19) reduces to

$$
J\left(w^{(n)}(\xi)\right)=\tilde{\lambda_{n}} w^{(n)}(\xi),
$$

where $\lambda_{n}=-\ln N \tilde{\lambda_{n}}$. Equation (25) is the differential equation fulfilled by the Jacobi polynomials $P_{\ell}^{1,1}(\bar{x})$ at the eigenvalues [32]

$$
\tilde{\lambda_{n}}=-\ell(\ell+3)
$$

with $\ell=0,1, \ldots$ and $n=\ell+1$. After substitution of Eqs. (23)-(26) into Eq. (19), the eigenfrequencies of the axial excitations are found from Eqs. (15) and (16) and take the form

$$
\omega_{n}^{\| \mathrm{Jac}}=\nu \sqrt{\frac{n(n+1)}{2}} .
$$

Analogously, the eigenfrequencies of the transverse modes are obtained from Eq. (18) with Eq. (19), resulting in

$$
\omega_{n}^{\perp \mathrm{Jac}}=\sqrt{\nu_{t}^{2}-\frac{(n-1)(n+2)}{4} \nu^{2}} .
$$

It should be noted that the solutions of Eq. (23) for $\ell=0$ and $\ell=1$ are exact solutions of the original problem (14): The corresponding eigenmodes $w^{(1)}(x)=$ const (center of mass) and $w^{(2)}(x) \propto x$ (stretch mode), which are the continuum limits of the eigenmodes we have found for the discrete case, are in fact Jacobi polynomials. The result for the center of mass is obvious. The exact result for the stretch mode can be understood, noting that $P_{1}^{1,1}(\xi)$ has only one node, whose position coincides with the center of the chain $\xi=0$ and thus with the symmetry center for reflections. Hence, its position is independent of the number of ions in the chain, and in particular it is independent of whether the ions distribution is discrete or continuous.

It is remarkable that the dispersion relation (27) coincides with a specific one-dimensional limit of a three-dimensional continuum mean-field theory, like the one developed in [33], although there is no obvious justification for this. The two limiting cases - the uniform spheroidal fluid of [33] and the case of $N$ strongly coupled oscillators investigated in this work-seem to provide the same axial eigenfrequencies in the long-wavelength regime and in the limit $N \gg 1$. This result is intriguing, especially if put in connection with theory of cold gases in low dimensions, where different dispersion relations are obtained depending on the assumption on the type of mean-field interaction $[34,38]$ and will be the object of future investigations.

Figure 1 presents the comparison between the spectrum of eigenfrequencies obtained with several methods. The solid lines show the result for 1000 ions, obtained by numerically diagonalizing the matrix (5) in Eqs. (6)-(8) after calculating the equilibrium positions by numerically solving the set of equations (3). The grey line shows the results in Eqs. (27) and (28), which have been truncated, as they do not correctly reproduce the short-wavelength eigenmodes. The shortwavelength modes are better evaluated by using a more proper approximation for this regime. The dotted line gives the spectra evaluated using the method of Dyson [35], dis- 

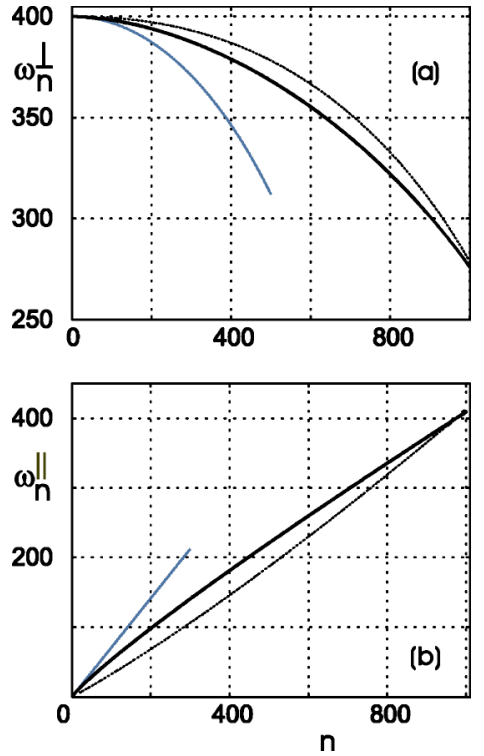

FIG. 1. (a) Transverse and (b) axial spectrum of eigenfrequencies (in units of $\nu$ ) for a chain of $N=1000$ ions and with $\nu_{t}=400 \nu$. The solid line corresponds to the numerical solution of Eqs. (6)-(8) with Eq. (3). The grey line shows (a) $\omega_{n}^{\perp \mathrm{Jac}}$, (b) $\omega_{n}^{\| \mathrm{Jac}}$ : These curves have been truncated, as they do not correctly reproduce the shortwavelength eigenmodes. The dotted line gives the spectra evaluated using the method of Dyson [35] implemented in Sec. III B.

cussed in Sec. III B and implemented in order to reproduce the density of states in the short-wavelength limit.

Figure 2 exhibits the part of the spectrum with the longwavelength axial modes: Here, one sees that Eq. (27) approximates well the lowest part of the axial spectrum, where the limit of continuous charge distribution is reasonable.

We remark that, apart from the first two eigenmodes, the Jacobi polynomials describe the eigenmode excitation at leading order in $\ln N$ and near the center of the chain, where the interparticle separation is of order $1 / N$ and the distribution of charges can be treated as a continuum for sufficiently long wavelengths. The continuum approximation fails at the edges, where the interparticle spacing is significantly larger and Eq. (12) is not meaningful. In particular, Eq. (13) gives the upper bound for the chain length, which would be obtained in the limit of $N \gg 1$ particles. Hence, a reasonable boundary condition is to assume that the eigemodes and their derivatives vanish at $x= \pm L$, where there are no charges and hence the energy density is zero. The solution (23) taken at the center of the chain neglects the charges at the edges on the basis of the observation that there the number of ions is much smaller than at the center and their contribution to the integral (17) can therefore be neglected.

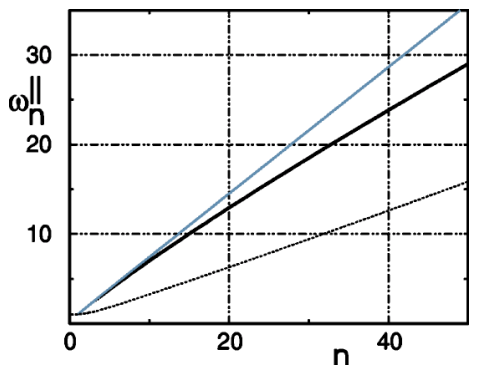

FIG. 2. Long-wavelength excitations of the axial spectrum of eigenfrequencies. Same notation and parameters as in Fig. 1.

The evaluation of the correction to the results (27) and (28) should be done in perturbation theory in the parameter $1 / \ln N$, following an analogous procedure to the one applied in [20] for evaluating the correction to the density of ions (12) and to the equilibrium length (13). In practice, this expansion has a very slow convergence and does not allow for a simple analytical expression. Nevertheless, the comparison with the spectrum evaluated numerically, by solving Eqs. (3) and (6)-(8), shows that Eqs. (27) and (28) give already a good estimate of the eigenfrequencies for a chain of ten ions, as can be seen in Fig. 3(a), where the relative deviation of the frequency given by Eq. (27) from the numerical result is plotted for chains with different number of ions. Figure 3(b) shows the shift $\omega_{n}^{\| \mathrm{Jac}}-\omega_{n}^{\|}$compared with the spectral distance between neighboring levels. The agreement is in general valid, respectively, for the axial low modes and the transverse high modes, and exhibits a very slow improvement as the number of ions in the chain increases, due to the slow convergence of the $1 / \ln N$ expansion.

\section{B. Density of states in the short-wavelength limit}

Simple physical considerations show that the shortwavelength eigenmodes are characterized by relatively large displacements of the ions around the center of the chain, while the ions at the edges nearly do not move. In fact, the interparticle distance is minimal in the middle of the chain, and for $N \gg 1$ it is of order $1 / N$, while it is consistently larger at the edges. Hence, a wave cannot propagate in a region where the interparticle spacing is larger than the wavelength. Here, we can make some simplifying assumptions in solving the eigenvalue equations (6)-(8) for the short-wavelength modes. In fact, in the center of the chain we expect that the relevant contributions to the force on an ion originate from the neighboring charges, as nearby groups of charges move in opposite directions, resulting in forces that mutually can-
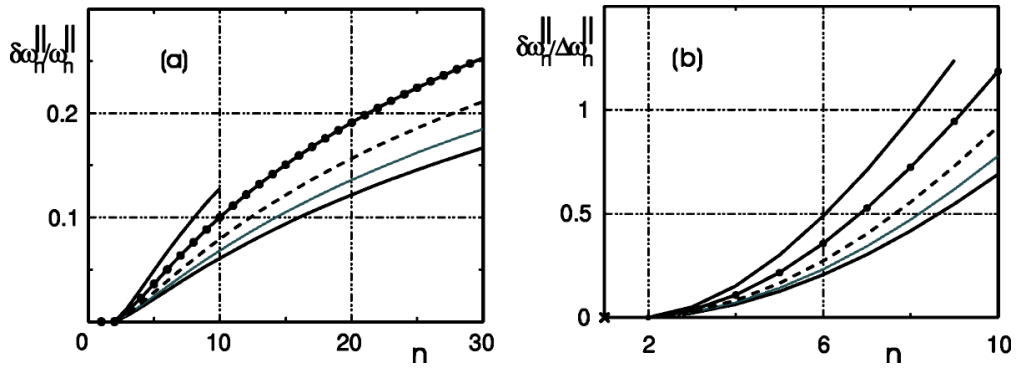

FIG. 3. (a) $\delta \omega_{n}^{\|} / \omega_{n}^{\|}$and (b) $\delta \omega_{n}^{\|} / \Delta \omega_{n}^{\|}$as a function of $n$, where $\delta \omega_{n}=\omega_{n}^{\| J \mathrm{Jac}}-\omega_{n}^{\|\|^{n}}$ and $\Delta \omega_{n}^{\|}$ $=\omega_{n+1}^{\|}-\omega_{n}^{\|}$. The frequencies $\omega_{n}^{\| n}$ are obtained by solving numerically Eq. (6) with Eq. (3). From top to bottom: $N=10,50,200,500,1000$. 
cel. By this hypothesis, in Eqs. (6)-(8) we keep only the nearest-neighbor interaction, so that the equations to solve are

$$
\begin{gathered}
\ddot{q}_{i}+\nu^{2} q_{i}=\Lambda\left[q_{i}, q_{i \pm 1}\right], \\
\ddot{y}_{i}+\nu_{t}^{2} y_{i}=-\frac{1}{2} \Lambda\left[y_{i}, y_{i \pm 1}\right], \\
\ddot{z}_{i}+\nu_{t}^{2} z_{i}=-\frac{1}{2} \Lambda\left[z_{i}, z_{i \pm 1}\right],
\end{gathered}
$$

where the tridiagonal matrix $\Lambda$ is defined by its action on the vector $\left(w_{1}, \ldots, w_{i-1}, w_{i}, w_{i+1}, \ldots, w_{N}\right)$ through

$$
\Lambda\left[w_{i}, w_{i \pm 1}\right]=\Lambda_{i}\left(w_{i+1}-w_{i}\right)+\Lambda_{i-1}\left(w_{i-1}-w_{i}\right)
$$

and $\Lambda_{i}=K_{i, i+1} / m$ where $K_{i, j}$ is given in Eq. (5). The matrix $\Lambda$ is symmetric, and we denote its characteristic frequencies by $-\widetilde{\omega}^{2}$. Following the derivation of Dyson [35], which is summarized in Appendix $\mathrm{B}$, the density of states $D\left(\widetilde{\omega}^{2}\right)$ for $N \gg 1$ is found from the characteristic function $\Omega(\theta)$ according to

$$
D(1 / z)=-z^{2} \operatorname{Re}\left[\frac{1}{\mathrm{i} \pi} \lim _{\epsilon \rightarrow 0} \frac{d \Omega}{d \theta}(-z+\mathrm{i} \epsilon)\right],
$$

while $\Omega(\theta)$ is explicitly evaluated by using the properties of antisymmetric matrices and takes the form

$$
\Omega(\theta)=\lim _{N \rightarrow \infty} \frac{1}{N} \sum_{j=1}^{2 N-1} \ln [1+\zeta(\theta, j)] .
$$

Here, $\zeta(\theta, j)$ is the infinite continued fraction,

$$
\zeta(\theta, j)=\frac{\theta \tilde{\Lambda}_{j}}{1+\frac{\theta \tilde{\Lambda}_{j+1}}{1+\frac{\theta \tilde{\Lambda}_{j+2}}{\ldots}}}=\frac{\theta \tilde{\Lambda}_{j}}{1+\zeta(\theta, j+1)}
$$

and $\widetilde{\Lambda}_{2 i-1}=\widetilde{\Lambda}_{2 i}=\Lambda_{i}$. For $N \gg 1$ and around the center of the chain we may assume $\Lambda(\xi)$ to be a slowly varying function of the position $\xi$, such that $\Lambda_{i+1}=\Lambda_{i}+\delta \Lambda_{i}$ and $\delta \Lambda_{i} / \Lambda_{i} \ll 1$. This allows us to evaluate explicitly $\zeta(\theta, j)$ at first order in $\delta \Lambda_{i}$. For this purpose, we define $\tilde{\Lambda}_{j-1}=\tilde{\Lambda}_{j}=\Lambda$ and $\tilde{\Lambda}_{j+1}$ $=\widetilde{\Lambda}_{j+2}=\Lambda+\delta \Lambda$, where $\delta \Lambda$ is the first-order variation. Furthermore, we denote $\zeta(\theta, j)=\zeta$ and assume $\zeta(\theta, j+2)=\zeta+\delta \zeta$, where $\delta \zeta$ is a first-order variation. We substitute these quantities into Eq. (35), keeping only the terms up to first order, and look for a consistent solution. The resulting equation is

$$
\delta \zeta(2 \zeta+1)=\theta \delta \Lambda
$$

which is integrated to

$$
\zeta^{2}+\zeta-\theta \Lambda=0
$$

Here, we have taken the integration constant to be zero since at the boundaries of the chain $\Lambda \rightarrow 0$. The resulting solution has the form

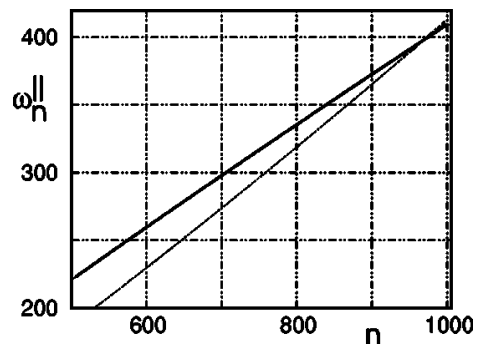

FIG. 4. Short-wavelength excitations of the axial spectrum of eigenfrequencies. Same notation and parameters as in Fig. 1.

$$
\zeta(\theta, \xi)=\frac{1}{2}[\sqrt{1+4 \theta \Lambda(L \xi)}-1],
$$

leading to the characteristic function

$$
\begin{aligned}
\Omega(\theta) & =\lim _{N \rightarrow \infty} \frac{2}{N} \sum_{j=1}^{N-1} \ln \left[\frac{1}{2}\left[\sqrt{1+4 \theta \Lambda\left(x_{j}\right)}+1\right]\right] \\
& =3 \int_{0}^{1} \mathrm{~d} \xi n(\xi) \ln \left[\frac{1}{2}[\sqrt{1+4 \theta \Lambda(L \xi)}+1]\right],
\end{aligned}
$$

where we have used the rescaled variable $\xi$ and the fact that the integrand is even in the interval $(-1,1)$. Equation (39) corresponds to the continuum limit of the discrete summation in Eq. (34), and it is valid away from the edges for $N \gg 1$. Substituting Eq. (39) into Eq. (33) we obtain the equation for the density of states as a function of the physical parameters,

$$
D(1 / z)=\frac{6 z}{\pi} \int_{0}^{f(z)} \mathrm{d} \xi n(\xi)\left[\frac{1}{\sqrt{-1+4 z \Lambda(L \xi)}}\right],
$$

where $f(z)=\sqrt{1-\left(1 / 4 \Lambda_{0} z\right)^{1 / 3}}$, while

$$
\Lambda(L \xi)=\Lambda_{0}\left(1-\xi^{2}\right)^{3},
$$

with

$$
\Lambda_{0}=\frac{\mathcal{K}_{0}}{a(\xi=0)^{3}} \nu^{2} \approx \frac{9}{32} \frac{N^{2}}{\ln N} \nu^{2}
$$

at leading order in $\ln N$.

Equation (40) with $\widetilde{\omega}^{2}=1 / z$ gives the density of states of the short-wavelength modes. The corresponding spectrum, evaluated by integrating the density of states (40), is compared with the numerical result in Fig. 1. The shortwavelength part of the spectrum is shown in Fig. 4. The deviations from the numerical result are due to the assumption of nearest-neighbor coupling and are small in the shortwavelength part of the spectrum, showing that Eq. (40) provides a good approximation in this regime. In particular, this result allows us to evaluate explicitly the value of the maximal axial frequency $\omega_{\max }^{\|}$, the minimal transverse frequency $\omega_{\text {min }}^{\perp}$, and the spectrum of the eigenfrequencies in their neighborhood. The maximal axial frequency and the minimal transverse frequency are found from the maximal value of $z$ for which the integrand in Eq. (40) is real, corresponding to $f(z)=0$. For larger values of $z$ the density of states vanishes. The corresponding eigenvalue of the matrix $\Lambda$ is $\widetilde{\omega}^{2}=4 \Lambda_{0}$. 


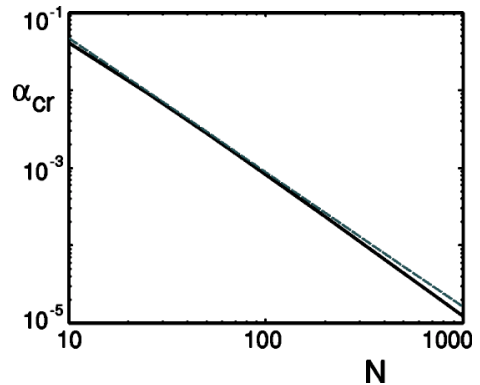

FIG. 5. $\alpha_{\text {cr }}$ as a function of the number of ions. The solid curve gives the result (46). The dashed curve is a fit according to the function $c N^{-1.73}$, with $c=2.53$, as calculated numerically in [23] (see also [24]).

From Eqs. (29)-(31) one finds $\omega_{\max }^{\|}=\sqrt{\nu^{2}+4 \Lambda_{0}}$ and $\omega_{\min }^{\perp}$ $=\sqrt{\nu_{t}^{2}-2 \Lambda_{0}}$. Therefore, the largest value of the axial frequency is determined by the largest value of the spring constant, which is the value of the spring constant at the center of the chain, and at leading order in $\ln N$ is

$$
\omega_{\max }^{\|} \approx \nu \sqrt{1+\frac{9}{8} \frac{N^{2}}{\ln N}} .
$$

Analogously, the smallest value of the transverse modes frequency is

$$
\omega_{\min }^{\perp} \approx \sqrt{\nu_{t}^{2}-\frac{9}{16} \nu^{2} \frac{N^{2}}{\ln N}} .
$$

From Eq. (44) one sees that $\omega_{\text {min }}^{\perp}$ can vanish for certain values of $\nu, \nu_{t}$, and $N$. We denote by

$$
\nu_{t, \mathrm{cr}}=\sqrt{\frac{9}{16} \nu^{2} \frac{N^{2}}{\ln N}}
$$

the value of the transverse frequency, such that for $\nu_{t}<\nu_{t, \mathrm{cr}}$ the linear chain is unstable with respect to excitations of the transverse vibrations. Using the notation introduced in [23] we define the critical value of the aspect ratio between the trap frequencies $\alpha_{\mathrm{cr}}=\nu^{2} / \nu_{t, \mathrm{cr}}^{2}$. It takes the value

$$
\alpha_{\mathrm{cr}}=\frac{16}{9} \frac{\ln N}{N^{2}},
$$

which fixes the condition on $\nu_{t}$ for the chain stability according to the inequality $\nu_{t}^{2}>\alpha_{\mathrm{cr}}^{-1} \nu^{2}$, and it is in agreement with the analytical estimate in [25], which was obtained under different requirements. In Fig. 5 we compare the result (46) with the relation $c N^{\beta_{1}}$, with $\beta_{1}=-1.73$ and $c=2.53$, obtained in [23] by fitting points calculated with molecular dynamics simulations and verified experimentally in [24]. Our result reproduces approximately this curve. At $\alpha_{\text {cr }}$ the crystal undergoes a structural transition from a linear string to a zigzag configuration, as has been observed in [6]. It is interesting to ask whether this structural transition can be considered as a phase transition, as discussed in [23-25]. A systematic investigation in this direction requires a proper definition of the thermodynamic limit for this kind of system. A natural thermodynamic limit, which will be discussed in the next section, is one where as $N \rightarrow \infty$, the axial frequency $\nu \rightarrow 0$ so that the density in the center is fixed. From Eqs. (12) and (13) this requires that the ratio $\nu^{2} N^{2} / \ln N$ be kept constant. From Eq. (45) it is clear that in this limit the critical transverse frequency tends to a well-defined value. The exploration of the properties of this transition in the thermodynamic limit will be object of further studies. Particularly interesting is the comparison with standard phase transitions [36].

\section{Phonon-like approximation}

It is natural to introduce a phonon-like approximation for the eigenmodes of Eqs. (6)-(8). In this approximation, a phonon-like solution is superimposed by a slowly varying amplitude, which takes into account the slow variation of the coupling strength $K_{i, j}$ of Eq. (5) as a function of both $i$ and $j$. This approximation, which is outlined in Appendix C, is reasonable for a relatively wide range of long-wavelength excitations compared to the Jacobi polynomials solution, discussed in Sec. III A (see Fig. 7 in Appendix C). It is inferior compared to the Jacobi polynomials in the very-longwavelength limit, and it is a bad approximation for the shortwavelength regime. Therefore, the phonon-like approximation, which is natural in condensed matter physics, is not an asymptotic approximation in the thermodynamic limit $N \rightarrow \infty$ both for the long-wavelength as well as for the shortwavelength parts of the spectrum.

\section{STATISTICAL MECHANICS}

In this section we use the density of states, which was evaluated in the preceding section, in order to derive the thermodynamic quantities of a linear crystal of $N$ ions. The linear chain is assumed to be in the regime of stability and to be in equilibrium with a thermal bath at temperature $T$. The oscillations around the classical equilibrium points are quantized using standard procedures [27]. It should be noted that in this limit the quantum statistics of the atoms is irrelevant: In fact, the single-particle wave packet is much smaller than the interparticle distance [37]. The dynamics of the crystal is thus described by $3 N$ harmonic oscillators of frequencies $\omega_{n}^{\|}$, $\omega_{n}^{\perp}$, where the frequencies $\omega_{n}^{\perp}$ are doubly degenerate. It is modeled by the Hamiltonian $\hat{H}$, obtained from $H$ after quantizing the eigenmodes of $H_{\text {har }}$ in Eq. (4). Here, $\hat{H}=H_{0}+\hat{H}_{n}$, where $H_{0}$ is the ground-state energy,

$$
H_{0}=V_{0}+\frac{\hbar}{2} \sum_{n=1}^{N}\left(\omega_{n}^{\|}+2 \omega_{n}^{\perp}\right),
$$

while $\hat{H}_{n}$ describes the contribution of the collective excitations,

$$
\hat{H}_{n}=\sum_{n=1}^{N} \hbar \omega_{n}^{\|} \hat{N}_{n}^{\|}+\sum_{n=1}^{N} \hbar \omega_{n}^{\perp}\left(\hat{N}_{n, y}^{\perp}+\hat{N}_{n, z}^{\perp}\right),
$$

where $\hat{N}_{n}^{\|}, \hat{N}_{n, y}^{\perp}$, and $\hat{N}_{n, z}^{\perp}$ are the operators counting the number of excitations. The term $V_{0}$ corresponds to the classical minimum energy of the Coulomb crystal. For an infinite chain, it is obtained by minimizing the classical energy with 
respect to the length $L$ using the density of charges in Eq. (12), and it is evaluated to be [20]

$$
V_{0}=\frac{3}{10} m \nu^{2} N L(N)^{2},
$$

where $L(N)$ is given in Eq. (13).

We remark that we investigate the thermodynamic quantities for crystals characterized by a finite number of particles $N$ and finite axial frequencies $\nu$-i.e., crystals of finite sizewhich may be close to experimental situations. It is, however, instructive to consider the definition of the thermodynamic limit for this kind of system characterized by strong correlations, where the effect of the charges at the edges cannot be neglected a priori in evaluating the statistical properties. Here, the thermodynamic limit can be appropriately defined by assuming constant interparticle spacing (thus constant linear density) at the center of the chain $x=0$-namely, requiring $a_{L}(0)$ to be constant. Denoting by $a_{0}=a_{L}(0)$, it scales as

$$
a_{0}=g\left(\frac{\sqrt{\ln N}}{\nu N}\right)^{2 / 3}
$$

where $g=\left(3 Q^{2} / m\right)^{1 / 3}$. This requirement corresponds to a vanishing axial frequency, according to $\nu \sim \sqrt{\ln N} / N$, as $N$ $\rightarrow \infty$. With this definition, in the thermodynamic limit the maximum axial frequency (43) and the critical transverse frequency (45) are independent of $N$ and of $\nu$, taking the values $\omega_{\max }^{\|}=3\left(g / 2 a_{0}\right)^{3 / 2}$ and $\nu_{t, \mathrm{cr}}=3 / 4\left(g / a_{0}\right)^{3 / 2}$. In the following, we derive the thermodynamic quantities for ion chains of finite size characterized by a fixed and finite value of the number of particles $N$ and of the axial frequency $\nu$, and discuss how these quantities behave when we take the specific thermodynamic limit that was defined above.

Assuming thermal equilibrium with the bath, the state of the system at constant number of ions $N$ is described by the density matrix of the canonical ensemble

$$
\rho=\frac{1}{Z} \exp (-\beta H)
$$

where $\beta=1 / k_{B} T$ and $Z$ is the partition function,

$$
Z=\exp \left(-\beta H_{0}\right) \prod_{n}\left[1-\exp \left(-\beta \hbar \omega_{n}\right)\right]^{-1},
$$

which determines the Helmholtz free energy

$$
F=-k_{B} T \ln Z .
$$

We identify the thermodynamic variables with $T$, the temperature, $N$, the number of atoms, and $\nu$, the axial trap frequency, whose variation corresponds to a variation in the length of the chain [1]. These are not a complete set of thermodynamic variables, but they fully determine the state of the crystal for the thermodynamic quantities we investigate in the following. In particular, we take $\nu_{t}$ as constant and assume $\omega_{\max }^{\|} \ll \omega_{\min }^{\perp}$, i.e., that there is a large gap between axial and transverse excitations. In this limit, we consider temperatures $T$ such that $k_{B} T \ll \hbar \omega_{\min }^{\perp}$. In this regime the transverse modes can be considered frozen; hence, the con- tribution due to their excitations to the crystal's thermodynamic properties can be neglected, and the dynamics of the system is one dimensional. The thermal energy of the crystal is given by

$$
U_{\mathrm{th}}=\left\langle\hat{H}_{n}\right\rangle=\sum_{n} \frac{\hbar \omega_{n}^{\|}}{\exp \left(\beta \hbar \omega_{n}^{\|}\right)-1} .
$$

The heat capacity $C_{a}=\partial U_{\mathrm{th}} /\left.\partial T\right|_{\nu, N}$ is

$$
C_{a}=\frac{\partial}{\partial T} \sum_{n} \frac{\hbar \omega_{n}^{\|}}{\exp \left(\beta \hbar \omega_{n}^{\|}\right)-1} .
$$

The behavior at high temperatures, such that $k_{B} T \gg \hbar \omega_{\max }^{\|}$ (but $k_{B} T \ll \hbar\left(\omega_{\min }^{\perp}\right)$, is given by the Dulong-Petit law $C_{a}=N k_{B}$ as is clear from Eq. (50). On the other hand, for $k_{B} T \ll \hbar \nu$ all modes are frozen and the energy of the chain is the zero-point energy $H_{0}$. For large number of particles $N \gg 1$ we can approximate the sum in Eq. (50) by the integral

$$
C_{a} \sim \frac{\partial}{\partial T} \int_{\nu}^{\omega_{\max }^{\|}} \mathrm{d} \omega g(\omega) \frac{\hbar \omega}{\exp (\beta \hbar \omega)-1},
$$

where $g(\omega)=\partial n / \partial \omega$ is the density of states. In particular, at temperatures such that the contribution of the longwavelength excitations to the sum is predominant and is given by Eq. (27), the resulting density of states is

$$
\left.g(\omega)\right|_{\text {low } T}=\frac{1}{\nu} \frac{4 \omega}{\sqrt{8 \omega^{2}+\nu^{2}}}
$$

and the heat capacity is given by the integral

$$
\left.C_{a}\right|_{\text {low } T} \sim \frac{\sqrt{2} k_{B}^{2}}{\hbar \nu} \frac{\partial}{\partial T} T^{2} \int_{x_{0}}^{\infty} \mathrm{d} x \frac{1}{\mathrm{e}^{x}-1} \frac{x^{2}}{\sqrt{x^{2}+x_{0}^{2} / 8}},
$$

where we have defined $x_{0}=\beta \hbar \nu$. Hence, the integrand and integration limits depend on the temperature through $x_{0}$. In this regime and for $k_{B} T \gg \hbar \nu$ we can set $x_{0} \sim 0$ in Eq. (51) and recover the result $C_{a}=\widetilde{c} T$, with $\tilde{c}=\sqrt{8} \zeta(2) k_{B}^{2} / \hbar \nu$, where $\zeta(2)$ is Riemann's zeta function. Therefore, for the considered regime the heat capacity is proportional to the temperature, which is a characteristic behavior encountered in a onedimensional Debye crystal [31]. In Fig. 6(a) the specific heat $c_{a}=C_{a} / N$ for $N=1000$ ions is plotted as a function of the temperature $T$. In the inset, the low-temperature behavior is shown and compared with the curve $\widetilde{c} T / N$ estimated above. The figure shows that the evaluated behavior, valid in the asymptotic limit of an infinite number of ions, provides a reasonable description of the specific heat at low temperatures.

It is remarkable that the heat capacity in Eq. (51) scales like $C_{a} \sim 1 / \nu$. The specific heat per particle $c_{a}=C_{a} / N$ behaves thus like $c_{a} \sim 1 / N \nu$ at low temperatures, and in the thermodynamic limit it vanishes as $c_{a} \sim 1 / \sqrt{\ln N}$. It thus depends on the number of ions, and this is a manifestation of the deviation from extensivity of the system's behavior. Note that the relative energy fluctuations are of the order $(\sqrt{\ln N} / N)^{1 / 2}$; therefore, the usual equivalence of ensembles holds.

The pressure $P$ in the axial direction is defined as 

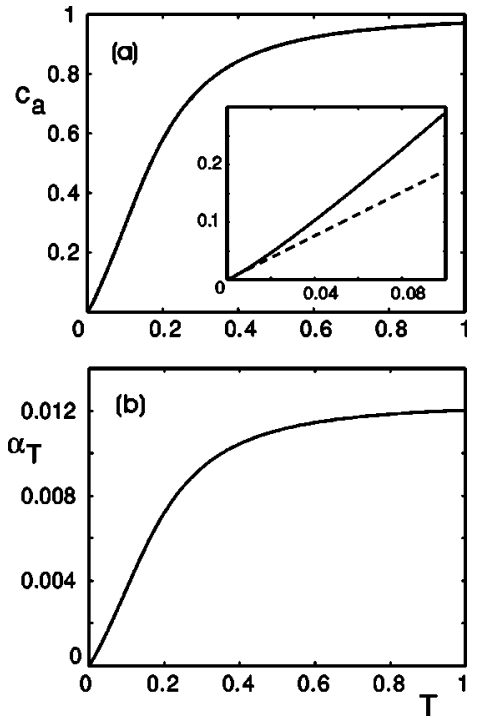

FIG. 6. (a) Specific heat $c_{a}=C_{a} / N$, in units of $k_{B}$, and (b) coefficient of thermal expansion $\alpha_{T}$, in arbitrary units, as a function of $T$, in units of $\hbar \omega_{\max }^{\|} / k_{B}$. Here, $N=1000$ and $\omega_{\max }^{\|} \ll \omega_{\min }^{\perp}$. This would correspond to an experiment with $\nu=2 \pi \times 1 \mathrm{kHz}$ and $\nu_{t}$ $=2 \pi \times 4 \mathrm{MHz}$. For these parameters and berillium atoms, $T$ is in units of the Debye temperature $\Theta_{D}$, with $\Theta_{D}=\hbar \omega_{\text {max }}^{\|} / k_{B} \approx 20 \mu \mathrm{K}$, and $\alpha_{T}$ in units of $10^{-5} \mu \mathrm{K}^{-1}$. The inset of (a) shows the lowtemperature behavior of the specific heat, and the dashed line is the estimated slope, with $\tilde{c}=\sqrt{8} \zeta(2) k_{B}^{2} / \hbar \nu$ (see text).

$$
P=-\left.\frac{\partial F}{\partial L}\right|_{T, N}
$$

and it is the variation of the free energy with the length of the string at constant $N$ and $T$. Under these conditions, the length of the crystal is varied by changing the axial trap frequency $\nu$, according to

$$
\left.\frac{\partial L}{\partial \nu}\right|_{T, N}=-\frac{2 L}{3 \nu},
$$

as obtained from Eq. (13). Substituting the explicit form of the free energy into Eq. (52), we find

$$
P=P_{0}+P_{T},
$$

where

$$
P_{0}=-\left.\frac{\partial H_{0}}{\partial L}\right|_{T, N}=\frac{V_{0}}{L}+\frac{3 \hbar}{4 L} \sum_{n}\left[\omega_{n}^{\|}+2 \omega_{n}^{\perp}\left(1-\frac{\nu_{t}^{2}}{\omega_{n}^{\perp 2}}\right)\right]
$$

is the pressure at zero temperature and $P_{T}$ gives the contribution of the excitations,

$$
P_{T}=\frac{3}{2 L} U_{\text {th }}
$$

In deriving Eqs. (54) and (55) we have used $\partial V_{0} / \partial L=$ $-V_{0} / L$, which results from Eq. (47), and the relations

$$
\frac{\partial}{\partial \nu} \omega_{n}^{\|}=\frac{\omega_{n}^{\|}}{\nu}
$$

$$
\frac{\partial}{\partial \nu} \omega_{n}^{\perp}=\frac{\omega_{n}^{\perp}}{\nu}\left[1-\left(\frac{\nu_{t}}{\omega_{n}^{\perp}}\right)^{2}\right],
$$

which are obtained from the functional dependence of $\omega_{n}^{\|}$ and $\omega_{n}^{\perp}$ on $\nu$, as can be extracted from Eqs. (16) and (18). Note that a variation of the axial trap frequency implies also a variation of the transverse eigenfrequencies, which give a contribution of opposite sign to the pressure, as is obvious from Eq. (54). However, the contribution due to the quantum-mechanical zero-point energy is very small compared to the classical term $V_{0} / L$. Therefore, $P_{0}$ is dominated by $V_{0} / L$ and, using Eq. (47), in the thermodynamic limit it scales as $P_{0} \sim \ln N$. The term $P_{T}$ depends on the temperature. For low temperatures, such that $k_{B} T \gg \hbar \nu$, it scales as $P_{T}$ $\sim 1 / L \nu \sim 1 / \sqrt{\ln N}$. At high temperatures, in the Dulong-Petit regime, $P_{T}$ depends on neither $N$ nor $\nu$. In the regime where the chain is thermally stable, which we consider here, $V_{0} \gg U_{\text {th }}$, giving $P \approx P_{0}$. Thus, the pressure is dominated by the zero-temperature contribution and in the thermodynamic limit $P \approx P_{0} \sim \ln N$. A useful relation for the following discussion is

$$
\left.\frac{\partial P}{\partial T}\right|_{L, N}=\frac{3}{2 L} C_{a} .
$$

The isothermal compressibility $\kappa_{T}$ is evaluated from the pressure according to

$$
\frac{1}{\kappa_{T}}=B=-\left.L \frac{\partial P}{\partial L}\right|_{T, N},
$$

where $B$ is the bulk modulus. Using Eqs. (54) and (55) in Eq. (57) we find

$$
\kappa_{T}=\left[-L \frac{\partial P_{0}}{\partial L}+\frac{3}{4 L}\left(5 U_{\mathrm{th}}-3 C_{a} T\right)\right]^{-1} .
$$

In the thermodynamic limit the bulk modulus $B$ is dominated by the zero-temperature contribution $-L \partial P_{0} / \partial L$, which in turn is dominated by the term $-L \partial V_{0} / \partial L \sim V_{0} / L \sim \ln N$. Therefore, $B \sim \ln N$ and the compressibility $\kappa_{T}$ vanishes as $1 / \ln N$.

The coefficient of thermal expansion $\alpha_{T}$ can be evaluated from knowledge of $\kappa_{T}$ and $C_{a}$ according to [31]

$$
\alpha_{T}=\left.\frac{1}{L} \frac{\partial L}{\partial T}\right|_{P, N}=-\frac{1}{L} \frac{\partial P /\left.\partial T\right|_{L}}{\partial P /\left.\partial L\right|_{T}}=\frac{3}{2 L} \kappa_{T} C_{a},
$$

where we have used Eq. (56). Since the compressibility is dominated by the zero-temperature term, the behavior of the coefficient of thermal expansion $\alpha_{T}$ as a function of $T$ is determined by the heat capacity: linear dependence at low temperatures and saturation at high temperatures. At low temperatures $C_{a} \sim 1 / \nu$, and as the thermodynamic limit is approached $\alpha_{T} \sim(\ln N)^{-3 / 2}$. At higher temperatures, when the heat capacity manifests the Dulong-Petit behavior $C_{a}=N k_{B}$, the coefficient of thermal expansion vanishes like $\alpha_{T} \sim 1 / \ln N$. In Fig. 6(b) the coefficient of thermal expansion for a chain of 1000 ions is presented. Calculations made with different numbers of ions, taking the trap frequency $\nu$ such that the linear density at the center of the chain remains 
constant, show that $\alpha_{T}$ decreases with $N$. The numerical results are consistent with the behavior we expect in different temperature regimes, according to the above considerations. In particular, for finite $N$ it is significantly different from zero. This is in contrast to the behavior found for uniform harmonic solids, where the coefficient of thermal expansion vanishes [31].

The thermodynamic quantities of a linear string of charges confined by a harmonic trap are affected by the way the thermodynamic limit is taken. Nevertheless, the behavior of the system is intrinsically nonextensive. The nonextensitivity is due to the strong correlation and the dimensionality of the crystal, which determine a regime where the correlation energy, associated with the discreteness of the individual charges, cannot be neglected [20]. It manifests in particular in the $\ln N$ terms appearing in the thermodynamic quantities. A representative example is the dependence of the specific heat per particle on $N$.

We finally remark on the thermal stability of the chain. The derivation presented in this section, in fact, relies on the assumption that the thermal excitations do not affect the stability of the system and thus the validity of the physical model we are considering-namely, of ions oscillating around their equilibrium positions. This condition is equivalent to the statement that the thermal energy at the considered temperatures is considerably lower than the equilibrium energy, and the displacements are much smaller than the respective interparticle distance. Hence, this condition is valid when

$$
\frac{Q^{2}}{a_{0}} \ln N \gg k_{B} T .
$$

This relation is amply satisfied for the parameters of the numerical evaluation of the thermodynamic quantities considered in this section.

\section{SUMMARY AND CONCLUSIONS}

We have investigated the dynamics of a linear crystal excitations with Coulomb interaction and in the presence of an external potential. We have derived an analytical formula for the density of states at long and short wavelengths. In the long-wavelength part of the spectrum, we have calculated analytically the eigenmodes and eigenfrequencies. The eigenmodes and eigenfrequencies for the center of mass and the first excitation of the axial and of the transverse motion are exact and independent of the number of ions. Apart for these modes, the results we derive are valid in the limit of an infinite number of ions. Nevertheless, they already give a good description of the spectrum of excitations of chains of dozens of ions. Using our results we study the statistical mechanics and thermodynamics of the linear chain.

Our derivation allows us to find an analytical formula for the critical transverse frequency required for determining the stability of the linear chain. It agrees with the analytical estimate by [25], which was obtained under different assumptions, and it is consistent with the formula fitted from numerical data [23] and verified experimentally [24]. It was suggested that this instability of the ion chain, resulting in a transition from a linear to a zigzag equilibrium configuration, can be treated as a phase transition [23,24]. In future works we will explore, using the formulation developed in this work, whether the thermodynamic quantities exhibit singularities characteristic of phase transitions [36]. This system differs from systems that are traditionally studied in the framework of statistical physics, since it is not extensive. In particular the specific heat per particle depends on the number of ions.

The results presented in this work show a statistical mechanics approach applied to a strongly correlated mesoscopic system. They contribute to ongoing research on lowdimensional cold gases $[38,39]$ and may be relevant to studies of the quantum dynamics of many-particle Coulomb systems like ion crystals in storage rings [9] and cold neutral plasmas $[40,41]$, which at sufficiently low temperatures are predicted to crystallize [42]. The connection with Wigner crystals, where the quantum statistics may play a relevant role [43], will be explored.

Moreover, the spectra of excitations here evaluated are relevant for implementations of quantum logic with ion traps, where knowledge of the long-wavelength modes is important for the realization of logic gates [16-19], as well as realization of solid-state models [44].

\section{ACKNOWLEDGMENTS}

It is our great pleasure to thank Andreas Buchleitner for hospitality at the Max-Planck-Institut für Physik komplexer Systeme in Dresden, where most of the research was done. S.F. is grateful to Wolfgang Schleich and the department of quantum physics in Ulm for hospitality. G.M. acknowledges the kind hospitality of the Institute for Theoretical Physics at Technion in Haifa. The authors acknowledge stimulating discussions and helpful comments of T. Antonsen, M. Drewsen, J. Eschner, D. Habs, P. Kienle, E. Ott, R. Prange, T. Schätz, J. Schiffer, E. Shimshoni, and H. Walther. This work was partly supported by the U.S. Israel Binational Science Foundation (BSF), by the Minerva Center of Nonlinear Physics of Complex Systems, by the Institute of Theoretical Physics at the Technion, by the Shlomo Kaplansky Academic Chair, and by the EU-networks QUEST and QGATES.

\section{APPENDIX A: SECULAR EQUATION OF THE LONG-WAVELENGTH EIGENMODES}

In this appendix we outline the derivation of the differential equation (22) and (23) from Eq. (17) and estimate the correction to the solution we find. By integration by parts, Eq. (17) takes the form

$$
I=I_{0}+I_{1}+I_{2},
$$

where

$$
\begin{aligned}
I_{0}= & \frac{1}{2 a(\xi)^{2}}\left[f_{\xi}(\xi-a)+f_{\xi}(\xi+a)\right] \\
& -\frac{1}{2 a(\xi)}\left[f_{\xi}^{\prime}(\xi-a)-f_{\xi}^{\prime}(\xi+a)\right] \\
& -\frac{1}{2} \ln a(\xi)\left[f_{\xi}^{(2)}(\xi-a)+f_{\xi}^{(2)}(\xi+a)\right],
\end{aligned}
$$




$$
\begin{aligned}
I_{1}= & -\frac{1}{2}\left(\frac{f_{\xi}(-1)}{(1+\xi)^{2}}+\frac{f_{\xi}(1)}{(1-\xi)^{2}}\right) \\
+ & \frac{1}{2}\left(\frac{f \xi^{\prime}(-1)}{1+\xi}-\frac{f_{\xi}^{\prime}(1)}{1-\xi}\right) \\
+ & \frac{1}{2}\left[f_{\xi}^{(2)}(-1) \ln (1+\xi)+f_{\xi}^{(2)}(1) \ln (1-\xi)\right], \\
I_{2}= & \frac{1}{2}\left[\int_{-1}^{\xi-a(\xi)} \mathrm{d} \xi^{\prime} \ln \left(\xi-\xi^{\prime}\right) f_{\xi}^{(3)}\left(\xi^{\prime}\right)\right. \\
& \left.-\int_{\xi+a(\xi)}^{1} \mathrm{~d} \xi^{\prime} \ln \left(\xi^{\prime}-\xi\right) f_{\xi}^{(3)}\left(\xi^{\prime}\right)\right],
\end{aligned}
$$

and $f_{\xi}\left(\xi^{\prime}\right)=n\left(\xi^{\prime}\right)\left[w(\xi)-w\left(\xi^{\prime}\right)\right]$. Clearly the integral $I_{0}$ results of the contribution of the ions around the ion at $\xi$. For longwavelength modes and away from the end-points $\xi= \pm 1$, the interparticle spacing $a(\xi)$ scales as $1 / N$ and it can be treated as an infinitesimal quantity. Expanding Eq. (A1) in $a(\xi)$ and keeping the leading order, we obtain Eq. (21). The integral (A2) contains the contribution of the end-points of the chain. Also, the integral (A3) is dominated by the end-points, as can be seen by integrating repeatedly by parts, taking the indefinite integral of $\ln x$, and making the reasonable assumption that $f(x)$ is an analytic function on the interval ( $-1,1)$.

By imposing the requirement that the density of ions and its derivatives vanish at the end-points, while $w(\xi)$ and its derivatives are bounded, the term $\Delta I=I_{1}+I_{2}$ is much smaller than $I_{0}$. In particular, the contributions at the end-points vanish, whereas the contributions to $I_{2}$ from the ions around the point $\xi$ are of order $a(\xi) \sim 1 / N$.

\section{APPENDIX B: CHARACTERISTIC FUNCTION FOR THE SHORT-WAVELENGTH SPECTRUM}

In this appendix we discuss the evaluation of the characteristic function for the eigenvalue problem in Eq. (29) following the treatment developed by Dyson [35]. The procedure can be immediately generalized to the eigenvalues of the transverse motion. Substituting $q_{j}(t)=\int \mathrm{d} \omega \mathrm{e}^{\mathrm{i} \omega t} \widetilde{q}_{j}(\omega) / 2 \pi$ into Eq. (29) and replacing $\tilde{q}_{i}(\omega)$ with $q_{i}$, we obtain

$$
-\left(\omega^{2}-\nu^{2}\right) q_{j}=\Lambda_{j}\left(q_{j+1}-q_{j}\right)+\Lambda_{j-1}\left(q_{j-1}-q_{j}\right) .
$$

The roots $-\widetilde{\omega}^{2}$ of the matrix $\Lambda$ are related to the eigenfrequencies $\omega$ by

$$
\omega=\sqrt{\widetilde{\omega}^{2}+\nu^{2}} .
$$

These roots can be found by solving the second-order differential equation

$$
\ddot{\bar{q}}_{j}=\Lambda_{j}\left(\bar{q}_{j+1}-\bar{q}_{j}\right)+\Lambda_{j-1}\left(\bar{q}_{j-1}-\bar{q}_{j}\right),
$$

where $\bar{q}_{j}(t)=\int \mathrm{d} \widetilde{\omega} \mathrm{e}^{\mathrm{i} \widetilde{\omega} t} q_{j}(\widetilde{\omega}) / 2 \pi$. Following [35], this problem reduces to the diagonalization of an antisymmetric matrix. In what follows we review the fundamental steps. We define the variables $s_{1}, \ldots, s_{N-1}$, such that $\dot{s}_{j}=\sqrt{\Lambda_{j}}\left(\bar{q}_{j+1}-\bar{q}_{j}\right)$. After sub- stituting into Eq. (B2) and integrating, we get the first-order differential equation $\dot{\bar{q}}_{j}=\sqrt{\Lambda_{j}} s_{j}-\sqrt{\Lambda_{j-1}} s_{j-1}$. We introduce the variables $u_{1}, \ldots, u_{2 N-1}$, such that $u_{2 j-1}=\bar{q}_{j}, u_{2 j}=s_{j}$, and the new matrix elements $\tilde{\Lambda}_{j}$, such that $\tilde{\Lambda}_{2 j}=\tilde{\Lambda}_{2 j-1}=\Lambda_{j}$. With these definition, we have now a set of $2 N-1$ first-order differential equations

$$
\dot{u}_{j}=\sqrt{\tilde{\Lambda}_{j}} u_{j+1}-\sqrt{\widetilde{\Lambda}_{j-1}} u_{j-1},
$$

which are now defined by the $(2 N-1) \times(2 N-1)$ antisym-

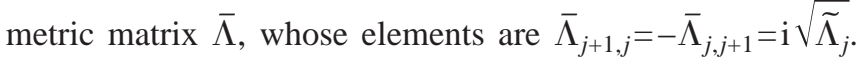
The characteristic frequencies $\widetilde{\omega}_{j}$ of the chain are the square roots of the characteristic roots of $-\Lambda$, of which one is 0 and the other $2 N-2$ come in pairs $\pm \widetilde{\omega}_{j}$.

Using the matrix $\bar{\Lambda}$, in the limit $N \gg 1$ the spectrum of characteristic frequencies $M\left(\widetilde{\omega}^{2}\right)$, which is defined as the proportion of roots with $\widetilde{\omega}_{j}^{2}<\widetilde{\omega}^{2}$, and the corresponding density $D\left(\widetilde{\omega}^{2}\right)=d M(\mu) /\left.d \mu\right|_{\mu=\widetilde{\omega}^{2}}$ can be derived from the characteristic function $\Omega(\theta)$, which is defined as [35]

$$
\Omega(\theta)=\lim _{N \rightarrow \infty} \frac{1}{2 N-1} \sum_{j=1}^{2 N-1} \ln \left(1+\theta \widetilde{\omega}_{j}^{2}\right)
$$

where $\theta$ is a complex variable. The density of characteristic frequencies $D(\mu)$ and the spectrum $M(\mu)$ are found by using the properties of the analytic continuation of $\Omega(\theta)$,

$$
\operatorname{Re}\left[\frac{1}{\mathrm{i} \pi} \lim _{\epsilon \rightarrow 0} \Omega(-z+\mathrm{i} \epsilon)\right]=\int_{1 / z}^{\infty} \mathrm{d} \mu D(\mu)=1-M(1 / z),
$$

from which Eq. (33) is obtained.

\section{APPENDIX C: AN ANSATZ FOR THE SECULAR EQUATION: SLOWLY VARYING ENVELOPE}

In this appendix we discuss an ansatz based on the phononic solution for a translationally invariant crystal, where we assume that the envelope, superimposed on the amplitude and the phase of the phononic solution, varies slowly as a function of the position. This ansatz is supposed to be valid for long-wavelength excitations and chains with $N \gg 1$ atoms.

The exact solution of Eqs. (6)-(8) in the limit of uniform charge distribution with constant spacing $a, K_{i, j} / m=\kappa / \mid i$ $-\left.j\right|^{3}$ with $\kappa=2 Q^{2} / a^{3}$, is [31]

$$
q_{j} \sim \mathrm{e}^{\mathrm{i}(k j a-\omega t)} .
$$

Here, $\omega$ is the eigenfrequency, $k$ the wave vector, and the boundary conditions for the uniform chain are fixed so that $q_{N}=q_{0}, \quad$ giving $\mathrm{e}^{\mathrm{i} k N a}=1$. Hence, $k a=2 \pi l / N$ with $l=0,1, \ldots, N-1$. The dispersion relation is obtained by substituting Eq. (C1) into Eq. (6) with $K_{i, j} / m=\kappa /|i-j|^{3}$. If the interaction is determined by the nearest neighbors, while the interaction with the other ions is neglected, then the eigenfrequencies are given by 


$$
\sqrt{\omega^{2}-\nu^{2}}=2 \sqrt{\kappa}\left|\sin \frac{k a}{2}\right| .
$$

Note that $\omega$ depends on $k a$, which takes the values $k a=2 \pi l / N$.

We now construct from this solution an ansatz for the inhomogeneous chain, in the limit of slowly varying spring constant $K_{j, j+1}$ over the wavelength of the propagating perturbation. We assume thus the local solution

$$
q_{j}=A_{j} \mathrm{e}^{\mathrm{i}(k j a-\omega t)},
$$

where $k a$ is a constant; i.e., we have assumed $k_{j} x_{j}^{(0)}=j k a$. The nonlinear variation of the phase with the site is included in $A_{j}$. The ansatz (C3) assumes that it is possible to write the solution as the product of a slowly varying amplitude and phase, represented by the factor $A_{j}$, and a fast oscillating part, which is the exponential term in Eq. (C1). The validity of this assumption is checked later on.

By substituting Eq. (C3) into Eq. (6), keeping only the nearest-neighbor interaction, we obtain

$$
\left(\omega^{2}-\nu^{2}\right) A_{j}=\kappa_{j}\left(A_{j}-A_{j+1} \mathrm{e}^{\mathrm{i} k a}\right)+\kappa_{j-1}\left(A_{j}-A_{j-1} \mathrm{e}^{-\mathrm{i} k a}\right),
$$

where $\kappa_{j}=K_{j, j+1} / m$. We make use of the fact that $\kappa_{j}$ varies slowly with position, which allows us to expand $A_{j}$ and $\kappa_{j}$ in $\delta j$, of order unity, according to

$$
\begin{gathered}
A_{j \pm 1} \approx A \pm \delta A, \\
\kappa_{j} \approx \kappa+\Delta \kappa+\delta \kappa_{j} / 2, \\
\kappa_{j-1} \approx \kappa+\Delta \kappa-\delta \kappa_{j} / 2,
\end{gathered}
$$

where $\delta A=(\partial A / \partial j) \delta j$ with $\delta j= \pm 1$. The spring constant has been divided into three contributions: $\kappa$ is constant and fulfills Eq. (C2), $\Delta \kappa$ is also constant, and $\delta \kappa_{j}=\kappa^{\prime} \delta j$, with $\kappa^{\prime}=\partial \kappa_{j} / \partial j$, such that $\kappa+\Delta \kappa \gg \delta \kappa_{j}$. The chosen variation of $\kappa_{j}$ reflects the symmetry under reflection of the crystal, such that the ion at the center is characterized by equal couplings on both sides (which implies the condition $\delta \kappa=0$ ). The term $\Delta \kappa$ does not depend on $j$, and it is the correction to $\kappa$, the spring constant fulfilling the dispersion relation for the case of a uniform chain: $\Delta \kappa \approx \kappa_{j}-\kappa$. Substituting Eqs. (C5)-(C7) into Eq. (C4) yields

$$
4 \Delta \kappa A \sin ^{2} \frac{k a}{2}-2 \mathrm{i} \kappa \delta A \sin k a-2 \mathrm{i} A \delta \kappa \sin k a=0,
$$

where we have applied the dispersion relation (C2). Since there is only one zeroth-order term in $j$, it is evident that $\Delta \kappa=0$ : Thus, there is no zeroth-order correction to the spring constant $\kappa$ of the uniform chain, and its value in this order is accounted for by Eq. (C2). The first-order expansion gives

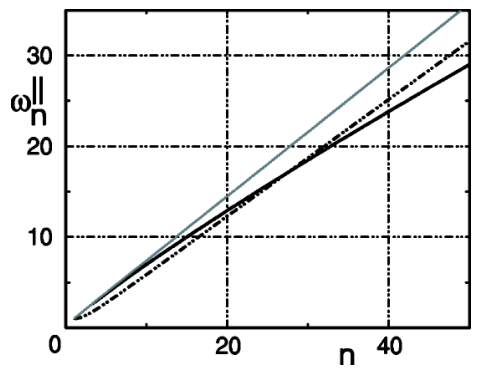

FIG. 7. Long-wavelength part of the axial eigenmodes spectrum for $N=1000$ ions. The dot-dashed line gives the spectrum evaluated using Eqs. (C10) and (C11), the solid line the numerical results, and the grey line the spectrum evaluated using Eq. (27).

thus a differential equation (valid for $\sin k a \neq 0$ )

$$
\frac{\delta A}{A}=-\frac{\delta \kappa}{2 \kappa},
$$

which admits the solution

$$
A_{j}=A_{0} \sqrt{\frac{\kappa_{0}}{\kappa_{j}}}
$$

The eigenmode has then the form

$$
q_{j}=\sqrt{\frac{\kappa_{0}}{\kappa_{j}}} \mathrm{e}^{\mathrm{i} k j a-\mathrm{i} \omega t},
$$

and the wave vector takes the same values as for a uniform crystal with half-periodic boundary conditions, taking into account the symmetry under reflection,

$$
k N a=\pi l,
$$

where $k=k(\omega)$ is given by Eq. (C2) and $l=0,1, \ldots, N-1$. Therefore, this ansatz gives simply a variation of the amplitude of the displacement of the ion as a function of the local spring constant, but no change in the phase, which is the same as the one of a uniform crystal. The amplitude is smaller at the center of the chain, where the ions are closer and the coupling constant is larger. Taking $\kappa_{0}=\Lambda_{0}$ as given by Eq. (42)-i.e., the maximum value of the spring constant in the chain - and using Eq. (C2), we obtain the maximal frequency as given in Eq. (43). A similar argument can be developed for the transverse frequency.

Figure 7 compares the prediction of the slowly varying ansatz with the numerical results and the Jacobi polynomials results given by Eq. (27). Here, it is obvious that the Jacobi polynomials provide a better approximation for the spectrum at the lowest eigenfrequencies. The curve evaluated from Eq. (C2) using Eqs. (C3) and (C11) lies close to the spectrum evaluated numerically for a larger range of modes, but it does not reproduce its asymptotic behavior for $N \rightarrow \infty$. 
[1] D. H. E. Dubin and T. M. O’Neil, Rev. Mod. Phys. 71, 87 (1999).

[2] P. K. Ghosh, Ion Traps (Clarendon Press, Oxford, 1995).

[3] F. Diedrich, E. Peik, J. M. Chen, W. Quint, and H. Walther, Phys. Rev. Lett. 59, 2931 (1987); R. Blümel, J. M. Chen, E. Peik, W. Quint, W. Schleich, Y. R. Shen, and H. Walther, Nature (London) 334, 309 (1988).

[4] D. J. Wineland, J. C. Bergquist, W. M. Itano, J. J. Bollinger, and C. H. Manney, Phys. Rev. Lett. 59, 2935 (1987).

[5] M. G. Raizen, J. M. Gilligan, J. C. Bergquist, W. M. Itano, and D. J. Wineland, Phys. Rev. A 45, 6493 (1992).

[6] G. Birkl, S. Kassner, and H. Walther, Nature (London) 357, 310 (1992); I. Waki, S. Kassner, G. Birkl, and H. Walther, Phys. Rev. Lett. 68, 2007 (1992).

[7] M. Drewsen, C. Brodersen, L. Hornekaer, J. S. Hangst, and J. P. Schiffer, Phys. Rev. Lett. 81, 2878 (1998); N. Kjargaard and M. Drewsen, ibid. 91, 095002 (2003).

[8] S. L. Gilbert, J. J. Bollinger, and D. J. Wineland, Phys. Rev. Lett. 60, 2022 (1988).

[9] T. Schätz, U. Schramm, and D. Habs, Nature (London) 412 , 717 (2001)

[10] Joseph N. Tan, J. J. Bollinger, B. Jelenkovic, and D. J. Wineland, Phys. Rev. Lett. 75, 4198 (1995).

[11] W. M. Itano, J. J. Bollinger, J. N. Tan, B. Jelenkovic, X.-P. Huang, and D. J. Wineland, Science 279, 686 (1998).

[12] P. Kienle, Naturwissenschaften 88, 313 (2001).

[13] N. Hermanspahn, H. Häffner, H. J. Kluge, W. Quint, S. Stahl, J. Verdù, and G. Werth, Phys. Rev. Lett. 84, 427 (2000); T. W. Koerber, M. H. Schacht, K. R. G. Hendrickson, W. Nagourney, and E. N. Fortson, ibid. 88, 143002 (2002).

[14] S. Bize, S. A. Diddams, U. Tanaka, C. E. Tanner, W. H. Oskay, R. E. Drullinger, T. E. Parker, T. P. Heavner, S. R. Jefferts, L. Hollberg, W. M. Itano, and J. C. Bergquist, Phys. Rev. Lett. 90, 150802 (2003); M. E. Poitzsch, J. C. Berquist, W. M. Itano, and D. J. Wineland, Rev. Sci. Instrum. 67, 129 (1996).

[15] K. Molhave and M. Drewsen, Phys. Rev. A 62, 011401(R) (2000).

[16] J. I. Cirac, P. Zoller, Phys. Rev. Lett. 74, 4091 (1995).

[17] A. Steane, Appl. Phys. B: Lasers Opt. 64, 623 (1997).

[18] F. Schmidt-Kaler, H. Häffner, M. Riebe, S. Gulde, G. P. T. Lancaster, T. Deuschle, C. Becher, C. F. Roos, J. Eschner, and R. Blatt, Nature (London) 422, 408 (2003).

[19] D. Leibfried, B. Demarco, V. Meyer, D. Lukas, M. Barrett, J. Britton, B. Jelenkovic, C. Langer, T. Rosenband, and D. J. Wineland, Nature (London) 422, 412 (2003).

[20] D. H. E. Dubin, Phys. Rev. E 55, 4017 (1997).
[21] J. P. Schiffer, J. Phys. B 36, 511 (2003).

[22] G. Morigi and S. Fishman, Phys. Rev. Lett. 93, 170602 (2004).

[23] J. P. Schiffer, Phys. Rev. Lett. 70, 818 (1993).

[24] D. G. Enzer, M. M. Schauer, J. J. Gomez, M. S. Gulley, M. H. Holzscheiter, P. G. Kwiat, S. K. Lamoreaux, C. G. Peterson, V. D. Sandberg, D. Tupa, A. G. White, R. J. Hughes, and D. F. V. James, Phys. Rev. Lett. 85, 2466 (2000).

[25] D. H. E. Dubin, Phys. Rev. Lett. 71, 2753 (1993).

[26] F. Dalfovo, S. Giorgini, L. P. Pitaevskii, and S. Stringari, Rev. Mod. Phys. 71, 463 (1999).

[27] D. F. V. James, Appl. Phys. B: Lasers Opt. 66, 181 (1998).

[28] D. Kielpinski, B. E. King, C. J. Myatt, C. A. Sackett, Q. A. Turchette, W. M. Itano, C. Monroe, D. J. Wineland, and W. H. Zurek, Phys. Rev. A 61, 032310 (2000).

[29] G. Morigi and H. Walther, Eur. Phys. J. D 13, 261 (2001).

[30] D. H. E. Dubin and J. P. Schiffer, Phys. Rev. E 53, 5249 (1996). The values of the first odd excitations (stretch modes) can be also analytically determined by assuming a continuous distribution of charges and applying scaling considerations. We are grateful to Sandro Stringari for showing to us this alternative derivation.

[31] N. W. Ashcroft and N. D. Mermin, Solid State Physics (Saunders College, Philadelphia, 1976).

[32] Handbook of Mathematical Functions, edited by M. Abramowitz and I. Stegun (Dover, New York, 1968).

[33] D. H. E. Dubin, Phys. Rev. Lett. 66, 2076 (1991).

[34] C. Menotti and S. Stringari, Phys. Rev. A 66, 043610 (2002).

[35] F. J. Dyson, Phys. Rev. 92, 1331 (1953).

[36] P. M. Chaikin and T. C. Lubensky, Principles of Condensed Matter Physics (Cambridge University Press, Cambridge, England, 1995).

[37] J. Yin and J. Javanainen, Phys. Rev. A 51, 3959 (1995).

[38] S. Stringari, Phys. Rev. A 58, 2385 (1998).

[39] H. Moritz, T. Stöferle, M. Köhl, and T. Esslinger, Phys. Rev. Lett. 91, 250402 (2004); L. Santos, G. V. Shlyapnikov, and M. Lewenstein, ibid. 90, 250403 (2003).

[40] T. F. Gallagher, P. Pillet, M. P. Robinson, B. Laburthe-Tolra, and M. W. Noel, J. Opt. Soc. Am. B 20, 1091 (2003).

[41] T. C. Killian, M. J. Lim, S. Kulin, R. Dumke, S. D. Bergeson, and S. L. Rolston, Phys. Rev. Lett. 86, 3759 (2001).

[42] T. Pohl, T. Pattard, and J. M. Rost, Phys. Rev. Lett. 92, 155003 (2004).

[43] H. J. Schulz, Phys. Rev. Lett. 71, 1864 (1993).

[44] D. Porras and J. I. Cirac, Phys. Rev. Lett. 92, 207901 (2004). 